\title{
On the Capacity of Multisource Non-Coherent Network Coding
}

\author{
Soheil Mohajer, Mahdi Jafari, Suhas N. Diggavi, Christina Fragouli \\ School of Computer and Communication Sciences \\ École Polytechnique Fédéral de Lausanne, Lausanne, Switzerland
}

Email: \{soheil.mohajer, mahdi.jafarisiavoshani, suhas.diggavi, christina.fragouli\}@epfl.ch

\begin{abstract}
We consider multisource non-coherent network coding, where multiple sources send information to one or multiple receivers. We prove that this is equivalent to a "subspace" channel, that takes subspaces as inputs and outputs. We then show that the rate of each individual receiver is upper bounded as $\delta_{i}\left(T-\delta_{1}-\delta_{2}\right)$, where $\delta_{i}$ is what we define to be the "dominating" dimension in the subspace codebook of source $i$, and $T$ is the "coherence" time of the network.
\end{abstract}

\section{INTRODUCTION}

We consider a network operating with network coding, where intermediate nodes send linear combinations, chosen uniformly at random, of their incoming packets. We are interested in the case where more than one sources insert information in the network, destined to one or more receivers. This is often the case in wireless networks, for example during operations such as topology discovery, or in applications such as sensor networks.

We assume that neither the sources nor the receivers have knowledge of the operations the intermediate nodes perform (noncoherent communication). For this model, and the case of a single source, use of subspace coding was proposed in [1], [3], and capacity bounds were investigated in [4], [5], [7], [8]. Algebraic code constructions for multiple sources were recently investigated in [2].

In this paper we derive the (asymptotic) capacity region for the case of two sources. We show that this region forms a polytope with a finite number of corner points. We also provide a simple achievability scheme.

The paper is organized as follows. Section II introduces our notation, and Section III our model and main results. Section V presents an outer bound on the achievable rates, and Section IV presents an achievability scheme that achieves the outer bound.

\section{NOTATION AND DEFINITIONS}

We here introduce the notation used in this paper. We use: - $a \doteq b$ for $a$ and $b$ functions of the size of a finite field $\mathbb{F}_{q}$ to imply that $\frac{\log _{q} a}{\log _{q} b}$ goes to one as the size of the finite field increases (similarly for $a \leq b$ ).

- For subspaces $\pi_{1}$ and $\pi_{2}, \pi_{1} \sqsubseteq \pi_{2}$ implies that $\pi_{1}$ is a subspace of $\pi_{2}$.

This work was supported by the Swiss National Science Foundation award PP002-110483, and by the EU project N-CRAVE FP7 ICT-2007-215252.
- For subspaces $\pi_{1}$ and $\pi_{2}, \pi_{1} \oplus \pi_{2}$ is the smallest subspace that contains both $\pi_{1}$ and $\pi_{2}$, namely,

$$
\pi_{1} \oplus \pi_{2}=\left\{v_{1}+v_{2} \mid v_{1} \in \pi_{1}, v_{2} \in \pi_{2}\right\} .
$$

- For a matrix $x,\langle x\rangle$ is the subspace spanned by rows of $x$.

Definition 1: Grassmannian and Gaussian number. The Grassmannian $\operatorname{Gr}(T, d)_{q}$ is the set of all $d$-dimensional subspaces of the $T$-dimensional space over a finite field $\mathbb{F}_{q}$, namely,

$$
\operatorname{Gr}(T, d)_{q} \triangleq\left\{\pi \sqsubseteq \mathbb{F}_{q}^{T}: \operatorname{dim}(\pi)=d\right\} .
$$

The cardinality of $\operatorname{Gr}(T, d)_{q}$ is the Gaussian number, namely,

$$
\mathcal{G}(T, d)_{q} \triangleq\left|\operatorname{Gr}(T, d)_{q}\right| \doteq q^{d(T-d)} .
$$

Definition 2: We define $\mathcal{S}(T, m)_{q}$ to be the set (sphere) of all subspaces of dimension at most $m$ in the $T$-dimensional space $\mathbb{F}_{q}^{T}$, namely

$$
\mathcal{S}(T, m)_{q} \triangleq \bigcup_{d=0}^{m} \operatorname{Gr}(T, m)_{q}=\left\{\pi \sqsubseteq \mathbb{F}_{q}^{T}: \operatorname{dim}(\pi) \leq m\right\} .
$$

The cardinality of $\mathcal{S}(T, m)_{q}$ equals

$$
s(T, d)_{q}=\left|\mathcal{S}(T, d)_{q}\right| \doteq \sum_{d=0}^{m} q^{d(T-d)} .
$$

Definition 3: We denote by $\psi\left(T, n, \pi_{d}\right)_{q}$ the number of different $n \times T$ matrices with elements in a field $\mathbb{F}_{q}$, such that their rows span a specific subspace $\pi_{d} \in \mathbb{F}_{q}^{T}$ of dimension $0 \leq d \leq \min (n, T)$.

For simplicity, we will drop the subscript $q$ in the previous definitions.

\section{Model And Main Results}

We consider a network with two transmitters (sources), a single receiver ${ }^{1}$, and operation in timeslots (or generations, in the network coding literature). In each timeslot, source $i$, $i=1,2$, inserts $m_{i}$ packets in the network, each of length $T$ and with elements over a finite field $\mathbb{F}_{q}$. The receiver observes

${ }^{1}$ From the multicasting theorem in network coding, the same results hold for the case of multiple receivers interested in the information from both sources. 
$n$ uniform at random combinations of these packets. Following [7], [8], we model the network operation at timeslot $l$ as a multiple access channel (MAC):

$$
Y[\ell]=H_{1}[\ell] X_{1}[\ell]+H_{2}[\ell] X_{2}[\ell] .
$$

Each $H_{i}, i=1,2$, is an $n \times m_{i}$ matrix, chosen uniformly at random over all possible matrices of appropriate dimensions, and i.i.d over different blocks. The packets that the sources insert in the network and the receiver observes are collected as rows of matrices $X_{1}, X_{2}$ and $Y$, respectively. Equivalently, each $X_{i}$ is a matrix chosen from $\widetilde{\mathcal{X}}_{i} \triangleq \mathbb{F}_{q}^{m_{i} \times T}$, the input alphabet of the $i$-th source, and $Y$ is a matrix from the set $\widetilde{\mathcal{Y}} \triangleq \mathbb{F}_{q}^{n \times T}$. We can think of $T$, the packet length, as the coherence time of the network.

For the channel in (2), the transition probability $P_{Y \mid X_{1}, X_{2}}$ can be written as [7]

$$
\begin{aligned}
\widetilde{P}_{\tilde{\mathcal{Y}} \mid \tilde{\mathcal{X}}_{1} \tilde{\mathcal{X}}_{2}}\left(y \mid x_{1}, x_{2}\right) \\
= \begin{cases}q^{-n \operatorname{dim}\left(\left\langle x_{1}\right\rangle \oplus\left\langle x_{2}\right\rangle\right)} & \langle y\rangle \sqsubseteq\left\langle x_{1}\right\rangle \oplus\left\langle x_{2}\right\rangle, \\
0 & \text { otherwise. }\end{cases}
\end{aligned}
$$

Note that

$\operatorname{dim}\left(\left\langle x_{1}\right\rangle \oplus\left\langle x_{2}\right\rangle\right)=\operatorname{dim}\left(\left\langle x_{1}\right\rangle\right)+\operatorname{dim}\left(\left\langle x_{2}\right\rangle\right)-\operatorname{dim}\left(\left\langle x_{1}\right\rangle \cap\left\langle x_{2}\right\rangle\right)$.

Our first result is that this channel is equivalent to a "subspace" channel, that has subspaces as inputs and outputs.

Lemma 1: The MAC channel $C_{m-M A C}$ described by (3) is equivalent to the MAC channel $C_{s-M A C}$ with input alphabets $\mathcal{X}_{i}=\mathcal{S}\left(T, m_{i}\right)$, for $i=1,2$, output alphabet $\mathcal{Y}=\mathcal{S}(T, n)$, and transition probability

$$
\begin{aligned}
& \operatorname{Pr}\left(Y=\pi_{y} \mid X_{1}=\pi_{1}, X_{2}=\pi_{2}\right) \\
&= \begin{cases}\psi\left(T, n, \pi_{y}\right) q^{-n \operatorname{dim}\left(\pi_{1} \oplus \pi_{2}\right)} & \pi_{y} \sqsubseteq \pi_{1} \oplus \pi_{2}, \\
0 & \text { otherwise. }\end{cases}
\end{aligned}
$$

The main contribution of this work is to derive the capacity region of this channel.

Theorem 1: The capacity region of the channel in (4) for $\frac{T}{2}>\max \left(m_{1}+m_{2}, n\right)$ is given by

$$
\mathcal{R}^{*} \triangleq \text { convex hull } \bigcup_{\left(d_{1}, d_{2}\right) \in \mathcal{D}^{*}} \mathcal{R}\left(d_{1}, d_{2}\right),
$$

such that

$$
\mathcal{R}\left(d_{1}, d_{2}\right) \triangleq\left\{\left(R_{1}, R_{2}\right): R_{i} \leq R_{i}\left(d_{1}, d_{2}\right), i=1,2\right\},
$$

where $R_{i}\left(d_{1}, d_{2}\right) \triangleq d_{i}\left(T-d_{1}-d_{2}\right)$ for $i=1,2$, and

$$
\begin{aligned}
\mathcal{D}^{*} \triangleq\left\{\left(d_{1}, d_{2}\right):\right. & 0 \leq d_{i} \leq \min \left(n, m_{i}\right), \\
& \left.0 \leq d_{1}+d_{2} \leq \min \left(n, m_{1}+m_{2}\right)\right\}
\end{aligned}
$$

The rate region $\mathcal{R}^{*}$ is shown in Fig. 1 for a particular choice of parameters. In the rest of the paper we prove Theorem 1.

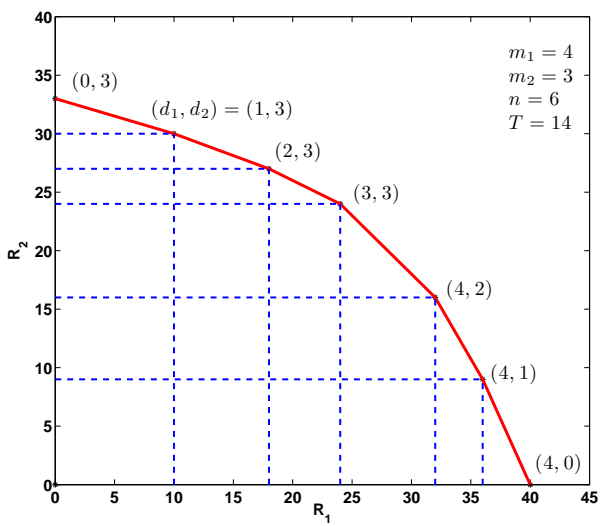

Fig. 1. The MAC region $\mathcal{R}^{*}$ for parameters $m_{1}=4, m_{2}=3, n=3$, $T=14$.

\section{ACHIEVABILity SCHEME}

In this section we illustrate a simple achievability scheme for the corner points of the rate region defined in Theorem 1. It is clear that other points in the rate region can be achieved using a time-sharing argument.

For given $\left(d_{1}, d_{2}\right) \in \mathcal{D}^{*}$, define the following codebooks.

$$
\begin{aligned}
& \tilde{\mathcal{C}}_{1} \triangleq\left\{\left\langle X_{1}\right\rangle: X_{1}=\right. \\
& {\left[\begin{array}{c|c|c}
\mathbf{I}_{d_{1} \times d_{1}} & \mathbf{0}_{d_{1} \times d_{2}} & \mathbf{U}_{1} \\
\hline \mathbf{0}_{\left(m_{1}-d_{1}\right) \times d_{1}} & \mathbf{0}_{\left(m_{1}-d_{1}\right) \times d_{2}} & \mathbf{0}_{\left(m_{1}-d_{1}\right) \times\left(T-d_{1}-d_{2}\right)}
\end{array}\right],} \\
& \left.\mathbf{U}_{1} \in \mathbb{F}_{q}^{d_{1} \times\left(T-d_{1}-d_{2}\right)}\right\}
\end{aligned}
$$

and

$$
\begin{aligned}
& \tilde{\mathcal{C}}_{2} \triangleq\left\{\left\langle X_{2}\right\rangle: X_{2}=\right. \\
& {\left[\begin{array}{c|c|c}
\mathbf{0}_{d_{2} \times d_{1}} & \mathbf{I}_{d_{2} \times d_{2}} & \mathbf{U}_{2} \\
\hline \mathbf{0}_{\left(m_{2}-d_{2}\right) \times d_{1}} & \mathbf{0}_{\left(m_{2}-d_{2}\right) \times d_{2}} & \mathbf{0}_{\left(m_{2}-d_{2}\right) \times\left(T-d_{1}-d_{2}\right)}
\end{array}\right],} \\
& \left.\mathbf{U}_{1} \in \mathbb{F}_{q}^{d_{2} \times\left(T-d_{1}-d_{2}\right)}\right\} .
\end{aligned}
$$

Transmitting messages from the codebooks, we have

$$
\begin{aligned}
Y & =H_{1} X_{1}+H_{2} X_{2} \\
& =\left[\tilde{H}_{1}\left|\tilde{H}_{2}\right| \tilde{H}_{1} \mathbf{U}_{1}+\tilde{H}_{2} \mathbf{U}_{2}\right],
\end{aligned}
$$

where $\tilde{H}_{i}$ is the first $d_{i}$ columns of $H_{i}$. Therefore, decoding at the receiver would be just construction of $\mathbf{U}_{1}$ and $\mathbf{U}_{2}$ having $\tilde{H}_{1} \mathbf{U}_{1}+\tilde{H}_{2} \mathbf{U}_{2}, \tilde{H}_{1}$, and $\tilde{H}_{2}$. Since $d_{1}+d_{2} \leq n$, the matrix $\left[\begin{array}{ll}\tilde{H}_{1} & \tilde{H}_{2}\end{array}\right]$ is full-rank with high probability, and therefore the decoder is able to decode $\mathbf{U}_{1}$ and $\mathbf{U}_{2}$.

Note that the achievability scheme is effectively the coding vectors approach [9]. This indicates that for $\frac{T}{2}>\max \left(m_{1}+\right.$ $\left.m_{2}, n\right)$ and $q$ large enough, the subspace coding and the coding vectors approach achieve the same rate. 


\section{OUter bound on the Admissible Rate Region}

The goal of this section is to show that the rate pair $\left(R_{1}, R_{2}\right)$ of two users over the channel described by (2) cannot be outside the region $\mathcal{R}^{*}$. We will give our proof in three steps. First, we find two upper bounds for $\mathcal{R}^{*}$ and then show that their intersection is in fact a subset of $\mathcal{R}^{*}$.

Let $\mathcal{R}_{\text {opt }}$ be the optimal rate region for the MAC channel (2). Then, by letting two transmitters to cooperate and using the result of [8] for the non-coherent single source channel we have the following lemma.

Lemma 2: We have $\mathcal{R}_{\text {opt }} \subseteq \mathcal{R}_{\text {coop }}$ where

$$
\mathcal{R}_{\text {coop }} \triangleq\left\{\left(R_{1}, R_{2}\right): R_{1}+R_{2} \leq k(T-k)\right\},
$$

and $k=\min \left(m_{1}+m_{2}, n\right)$.

In the rest of this section we will focus to derive another upper bound which is denoted by $\mathcal{R}_{\text {col }}$. This bound is based on the number of messages per channel use that each user can communicate over the multiple access channel provided that $n$ is large enough. It is clear that this assumption does not restrict the rate region, because the more number of packets the receiver receives, the better it can decode the messages.

For each time slot $t$, let $\mathcal{C}_{i}[t]$ be the codebook used by source $i$. For a single source scenario, we showed in [8] that we can use the set $\mathcal{S}(T, m)$ as our input alphabet for all time slots, and have the receiver successfully decode the sent messages. Thus the user can communicate $s(T, m)$ distinct messages. For the multi-source scenario $\mathcal{X}_{i}[t]$ is more restricted. This is because the transition probability of the channel $P_{Y \mid X_{1}, X_{2}}$ is of the form $P_{Y \mid X_{1} \oplus X_{2}}$. Thus, if $\left(X_{1}, X_{2}\right) \in \mathcal{X}_{1} \times \mathcal{X}_{2}$ and $\left(X_{1}^{\prime}, X_{2}^{\prime}\right) \in \mathcal{X}_{1} \times \mathcal{X}_{2}$ satisfy $X_{1} \oplus X_{2}=X_{1}^{\prime} \oplus X_{2}^{\prime}$, then the receiver cannot distinguish between them.

In the following we will bound the number of messages for each receiver in this case. In order to do so, we start with some useful definitions and lemmas. The proof of the lemmas is presented in the Appendix.

Definition 4: For a fixed $\pi_{1} \in \operatorname{Gr}\left(T, d_{1}\right)$, we define

$$
\mathcal{N}\left(\pi_{1}, d_{2}, \alpha\right) \triangleq\left\{\pi_{2} \in \operatorname{Gr}\left(T, d_{2}\right): \operatorname{dim}\left(\pi_{1} \cap \pi_{2}\right)=\alpha\right\} .
$$

Lemma 3: The cardinality of the set $N\left(\pi_{1}, d_{2}, \alpha\right)$ is given by

$$
n\left(d_{1}, d_{2}, \alpha\right)=\left|N\left(\pi_{1}, d_{2}, \alpha\right)\right| \doteq q^{\alpha\left(d_{1}-\alpha\right)+\left(d_{2}-\alpha\right)\left(T-d_{2}\right)} .
$$

Note that since $\left|N\left(\pi_{1}, d_{2}, \alpha\right)\right|$ depends on $\pi_{1}$ only through $d_{1}=\operatorname{dim}\left(\pi_{1}\right)$, we have replaced it by $d_{1}$.

Definition 5: For a fixed $\pi_{1} \in \operatorname{Gr}\left(T, d_{1}\right)$ and $\pi_{2} \in$ $\operatorname{Gr}\left(T, d_{2}\right)$, we define

$$
A\left(\pi_{1}, \pi_{2}\right) \triangleq\left\{\pi_{2}^{\prime} \in \operatorname{Gr}\left(T, d_{2}\right): \pi_{1} \oplus \pi_{2}^{\prime}=\pi_{1} \oplus \pi_{2}\right\} .
$$

Lemma 4: The cardinality of the set $A\left(\pi_{1}, \pi_{2}\right)$ is given by

$$
a\left(d_{1}, d_{2}, \alpha\right)=\left|A\left(\pi_{1}, \pi_{2}\right)\right| \doteq q^{d_{2}\left(d_{1}-\alpha\right)},
$$

where $\alpha=\operatorname{dim}\left(\pi_{1} \cap \pi_{2}\right)$. Note that since $\left|A\left(\pi_{1}, \pi_{2}\right)\right|$ only depends on the dimension of two subspaces and their intersection, we can express it as a function of $d_{1}, d_{2}$, and $\alpha$.
Definition 6: For a set $\mathcal{X} \subset \mathcal{S}(T, m)$, we denote the projection of $\mathcal{X}$ onto the set of $d$-dimensional subspaces by $\mathcal{X}(d)$. Formally,

$$
\mathcal{X}(d) \triangleq \mathcal{X} \cap \operatorname{Gr}(T, d)=\{X \in \mathcal{X}: \operatorname{dim}(X)=d\} .
$$

To communicate, each of our sources is going to use as alphabet a set of subspaces. For two such sets of subspaces $\mathcal{X}_{1}$ and $\mathcal{X}_{2}$, we can construct a table with $\left|\mathcal{X}_{1}\right|$ rows and $\left|\mathcal{X}_{2}\right|$ columns, each row (column) corresponding to one subspace $a$ (b) in $\mathcal{X}_{1}\left(\mathcal{X}_{2}\right)$. A coloring for this table is an assignment of colors to the cells of the table using a function col : $\mathcal{X}_{1} \times \mathcal{X}_{2} \rightarrow$ $\mathbb{N}$ such that $\operatorname{col}(a, b)=\operatorname{col}\left(a^{\prime}, b^{\prime}\right)$ if and only if $a \oplus b=a^{\prime} \oplus b^{\prime}$.

Theorem 2: For each uniquely decodable code $\mathcal{C}$ defined on some input alphabet $\mathcal{X}_{1} \times \mathcal{X}_{2}$ for a multiple access channel and for each time slot $t$, there exist integer numbers $0 \leq d_{i}(t) \leq$ $m_{i}$ such that

$$
\left|\mathcal{C}_{i}[t]\right| \dot{\leq} q^{\delta_{i}(t)\left(T-\delta_{1}(t)-\delta_{2}(t)\right)}, \quad i=1,2 .
$$

Here $\mathcal{C}_{i}[t]$ denotes the restriction of the code $\mathcal{C}_{i}$ to its $t$-th component.

Proof: We present the proof for a given fixed $t$, but sometimes drop the time index for brevity. For a fixed $t$, let $\delta_{i}$ be the dominating dimension in the set $\mathcal{X}_{i}$, i.e.,

$$
\delta_{i} \triangleq \arg \max _{d}\left|\mathcal{X}_{i}(d)\right|
$$

where, from definition $6, \mathcal{X}_{i}(d)$ contains all the d-dimensional subspaces in the codebook $X_{i}$. It is clear that

$$
\left|\mathcal{X}_{i}\right|=\sum_{d}\left|\mathcal{X}_{i}(d)\right| \leq m_{i}\left|\mathcal{X}_{i}\left(\delta_{i}\right)\right| \doteq\left|\mathcal{X}_{i}\left(\delta_{i}\right)\right| .
$$

By removing all subspaces from $\mathcal{X}_{1}\left(\mathcal{X}_{2}\right)$ except the ones that have dimension $\delta_{1}\left(\delta_{2}\right)$ we loose only a constant factor in the codebook size. Therefore the loss in the rate values would be negligible as $q$ grows. Consider the table constructed for $\mathcal{X}_{1}\left(\delta_{1}\right)$ and $\mathcal{X}_{2}\left(\delta_{2}\right)$. Let $\pi_{1} \in \mathcal{X}_{1}\left(\delta_{1}\right)$ be a $\delta_{1}$-dimensional subspace, and consider the corresponding row of the table. We further partition the columns of the table with respect to $\pi_{1}$ into $\bigcup_{\alpha=0}^{\min \left(\delta_{1}, \delta_{2}\right)} \mathcal{X}_{2}\left(\pi_{1}, \delta_{2}, \alpha\right)$, where

$$
\mathcal{X}_{2}\left(\pi_{1}, \delta_{2}, \alpha\right) \triangleq\left\{\pi_{2} \in \mathcal{X}_{2}\left(\delta_{2}\right): \operatorname{dim}\left(\pi_{1} \cap \pi_{2}\right)=\alpha\right\} .
$$

We use $K\left(\pi_{1}, \delta_{2}\right)$ and $K\left(\pi_{1}, \delta_{2}, \alpha\right)$ to denote the number of different colors in the row corresponds to $\pi_{1}$ and its intersection with $\mathcal{X}_{2}\left(\pi_{1}, \delta_{2}, \alpha\right)$, respectively. Note that $\mathcal{X}_{2}\left(\pi_{1}, \delta_{2}, \alpha\right)$ has at most $n\left(\delta_{1}, \delta_{2}, \alpha\right)$ elements, where each color appears $a\left(\delta_{1}, \delta_{2}, \alpha\right)$ times. Therefore the number of different colors in this partition can be upper bounded as

$$
K\left(\pi_{1}, \delta_{2}, \alpha\right) \leq \frac{n\left(\delta_{1}, \delta_{2}, \alpha\right)}{a\left(\delta_{1}, \delta_{2}, \alpha\right)} \doteq q^{\left(\delta_{2}-\alpha\right)\left(T-\delta_{1}-\delta_{2}+\alpha\right)} .
$$


As a result,

$$
\begin{aligned}
K\left(\pi_{1}, \delta_{2}\right) & \leq \sum_{\alpha=0}^{\min \left(\delta_{1}, \delta_{2}\right)} K\left(\pi_{1}, \delta_{2}, \alpha\right) \\
& \leq \sum_{\alpha=0}^{\min \left(\delta_{1}, \delta_{2}\right)} q^{\left(\delta_{2}-\alpha\right)\left(T-\delta_{1}-\delta_{2}+\alpha\right)} \\
& \doteq q^{\max _{0 \leq \alpha \leq \min \left(\delta_{1}, \delta_{2}\right)}\left(\delta_{2}-\alpha\right)\left(T-\delta_{1}-\delta_{2}+\alpha\right)} \\
& \doteq q^{\delta_{2}\left(T-\delta_{1}-\delta_{2}\right)}
\end{aligned}
$$

where the last asymptotic equality holds since $T \geq 2\left(\delta_{1}+\delta_{2}\right)$ and the exponent is a decreasing function of $\alpha$ for $0 \leq \alpha \leq$ $\min \left(\delta_{1}, \delta_{2}\right)$.

This argument holds for each choice of $\pi_{1}$. This means if user 1 transmits a $\delta_{1}$-dimensional subspace, the receiver cannot distinguish more that $q^{\delta_{2}\left(T-\delta_{1}-\delta_{2}\right)}$ different symbols. Thus the number of decodable messages user 2 can communicate is upper bounded by $q^{\delta_{2}\left(T-\delta_{1}-\delta_{2}\right)}$. The same argument holds for a fixed column $\pi_{2} \in \mathcal{X}_{2}$ which yields an upper bound to the number of communicated messages as $q^{\delta_{1}\left(T-\delta_{1}-\delta_{2}\right)}$.

From Theorem 2 we have the following corollary.

Corollary 1: We have $\mathcal{R}_{\text {opt }} \subseteq \mathcal{R}_{\text {col }}$ where

$$
\mathcal{R}_{\text {col }} \triangleq \text { convex hull } \bigcup_{\left(d_{1}, d_{2}\right) \in \mathcal{D}_{\text {col }}} \mathcal{R}\left(d_{1}, d_{2}\right) \text {, }
$$

and $\mathcal{D}_{\text {col }} \triangleq\left\{\left(d_{1}, d_{2}\right): 0 \leq d_{i} \leq m_{i}\right\}$.

Proof: Using Theorem 2, it is clear that the number of decodable pairs for each time instance is upper bounded by $\left(q^{R_{1}}, q^{R_{2}}\right)$ for some $\left(d_{1}, d_{2}\right) \in \mathcal{D}_{\text {col }}$. Coding over $L$ time instance can only provide rates which are convex combinations (time-sharing) of such $\left(R_{1}, R_{2}\right)$, for different values of $\left(d_{1}, d_{2}\right)$ used for different time slots. Therefore the rate pair cannot be outside of the region defined in the corollary.

By Lemma 2 and Corollary 1 we have already shown that $\mathcal{R}_{\text {opt }} \subseteq \mathcal{R}_{\text {coop }}$ and $\mathcal{R}_{\text {opt }} \subseteq \mathcal{R}_{\text {col }}$, respectively. Therefore $\mathcal{R}_{\text {opt }} \subseteq \mathcal{R}_{\text {coop }} \cap \mathcal{R}_{\text {col }}$. We have shown the achievability of $\mathcal{R}^{*}$ in Section IV, so it only remains to prove the following theorem.

Theorem 3: We have $\mathcal{R}_{\text {coop }} \cap \mathcal{R}_{\text {col }} \subseteq \mathcal{R}^{*}$.

Proof: Let $\left(R_{1}, R_{2}\right) \in \mathcal{R}_{\text {coop }} \cap \mathcal{R}_{\text {col }}$ be a corner point (note that since the convex hull is operating over a finite number of sets, we can still talk about corner points). It is clear that $\left(R_{1}, R_{2}\right)$ is either a corner point of $\mathcal{R}_{\text {col }}$, or is a middle point on the boundaries of $\mathcal{R}_{\text {col }}$ and $\mathcal{R}_{\text {coop }}$. For the former case, being a corner point of $\mathcal{R}_{\mathrm{col}}$, implies that it is of the form $\left(R_{1}, R_{2}\right)=\left(R_{1}\left(d_{1}, d_{2}\right), R_{2}\left(d_{1}, d_{2}\right)\right)$, for some $\left(d_{1}, d_{2}\right) \in \mathcal{D}_{\text {col. }}$. Also $\left(R_{1}, R_{2}\right) \in \mathcal{R}_{\text {coop }}$ implies

$$
R_{1}+R_{2}=\left(d_{1}+d_{2}\right)\left(T-\left(d_{1}+d_{2}\right)\right) \leq k(T-k),
$$

and therefore $d_{1}+d_{2} \leq k$, since $f(x) \triangleq x(T-x)$ is an increasing function for $x \in(0, T / 2)$. Hence $\left(d_{1}, d_{2}\right) \in \mathcal{D}^{*}$, and $\left(R_{1}, R_{2}\right) \in \mathcal{R}^{*}$. We claim that the second case never happens. The proof is given in Lemma 5 and Lemma 6.
Lemma 5: The set of corner points of $\mathcal{R}_{\mathrm{col}}$ is the set of all rate pairs of the form $\left(R_{1}, R_{2}\right)=\left(R_{1}\left(d_{1}, d_{2}\right), R_{2}\left(d_{1}, d_{2}\right)\right)$, for some $\left(d_{1}, d_{2}\right) \in \widetilde{\mathcal{D}}$, where

$$
\begin{aligned}
\widetilde{\mathcal{D}}=\{ & \left(0, m_{2}\right),\left(1, m_{2}\right), \ldots,\left(m_{1}, m_{2}\right), \\
& \left.\left(m_{1}, m_{2}-1\right), \ldots,\left(m_{1}, 1\right),\left(m_{1}, 0\right)\right\} .
\end{aligned}
$$

Proof: We will show that any point $\left(R_{1}\left(d_{1}, d_{2}\right), R_{2}\left(d_{1}, d_{2}\right)\right)$ is dominated by the segment connecting $\left(R_{1}\left(d_{1}+1, d_{2}\right), R_{2}\left(d_{1}+1, d_{2}\right)\right) \quad$ and $\left(R_{1}\left(d_{1}, d_{2}+1\right), R_{2}\left(d_{1}, d_{2}+1\right)\right)$. In order to show that, we have to prove that there exists some $\lambda \in[0,1]$, such that

$$
\begin{aligned}
& R_{1}\left(d_{1}, d_{2}\right)<\lambda R_{1}\left(d_{1}+1, d_{2}\right)+(1-\lambda) R_{1}\left(d_{1}, d_{2}+1\right), \\
& R_{2}\left(d_{1}, d_{2}\right)<\lambda R_{2}\left(d_{1}+1, d_{2}\right)+(1-\lambda) R_{2}\left(d_{1}, d_{2}+1\right) .
\end{aligned}
$$

After a little simplification, (14) can be rewritten as

$$
\begin{gathered}
\lambda\left[T-d_{1}-d_{2}-1\right]<d_{1}, \\
(1-\lambda)\left[T-d_{1}-d_{2}-1\right]<d_{2}, \\
\text { or } \quad \frac{d_{1}}{T-1-d_{1}-d_{2}}<\lambda<\frac{T-1-d_{1}-2 d_{2}}{T-1-d_{1}-d_{2}} .
\end{gathered}
$$

The last two inequalities can be satisfied for some choice of $\lambda$ if and only if $d_{1}+d_{2}<(T-1) / 2$. Therefore any $\left(d_{1}, d_{2}\right) \in$ $\mathcal{D}_{\text {col }}$ with $d_{1}<m_{1}, d_{2}<m_{2}$, and $d_{1}+d_{2}<(T-1) / 2$, cannot form a corner point, because otherwise $\left(d_{1}+1, d_{2}\right)$ and $\left(d_{1}, d_{2}+1\right)$ also belong to $\mathcal{D}_{\text {col }}$. Eliminating such $\left(d_{1}, d_{2}\right)$ from $\mathcal{D}_{\text {col }}$, we get $\widetilde{\mathcal{D}}$.

It is also easy to show that all of the rate pairs corresponding to $\left(d_{1}, d_{2}\right) \in \widetilde{\mathcal{D}}$ are on the boundary of $\mathcal{R}_{\text {col }}$. This can be done by comparing the slope of the connecting segment for two consecutive points (according to the order they are appeared in $\widetilde{\mathcal{D}}$ ). The slopes are

$$
\begin{gathered}
\mathcal{S}\left\{\left(R_{1}\left(t, m_{2}\right), R_{2}\left(t, m_{2}\right)\right) ;\left(R_{1}\left(t+1, m_{2}\right), R_{2}\left(t+1, m_{2}\right)\right)\right\} \\
=-\frac{m_{2}}{T-2 t-m_{2}-1} \quad \text { for } 0 \leq t \leq m_{1} \\
\mathcal{S}\left\{\left(R_{1}\left(m_{1}, t\right), R_{2}\left(m_{1}, t\right)\right) ;\left(R_{1}\left(m_{1}, t-1\right), R_{2}\left(m_{1}, t-1\right)\right)\right\} \\
\quad=-\frac{T-2 t-m_{1}-1}{m_{1}} \quad \text { for } 1 \leq t \leq m_{2} .
\end{gathered}
$$

It is easy to check that all the slopes are negative and they are in a decreasing order. Therefore, no point in the set $\widetilde{\mathcal{D}}$ can be an interior point.

Lemma 6: If $\mathcal{R}_{\text {col }} \nsubseteq \subseteq \mathcal{R}_{\text {coop }}$, then any intersecting point of $R_{1}+R_{2}=k(T-k)$ with the boundary of $\mathcal{R}_{\text {col }}$ is a point in the set

$$
\widetilde{\mathcal{D}} \cup\left\{\left(m_{1}-1,0\right), \ldots,(0,0),(0,1), \ldots,\left(0, m_{2}-1\right)\right\} .
$$

Proof: Note that $\mathcal{R}_{\text {col }} \nsubseteq \mathcal{R}_{\text {coop }}$ implies $m_{1}+m_{2}>n$. Since $\mathcal{R}_{\text {col }}$ is a convex region, its boundary intersect with the line $R_{1}+R_{2}=n(T-n)$ in exactly two points (it cannot be only one point, otherwise it would be inside of $\mathcal{R}_{\text {coop }}$ ). It is clear that the two corner points of $\mathcal{R}_{\text {col }}$, corresponding to $\left(d_{1}, d_{2}\right)=\left(\left(n-m_{2}\right)^{+}, \min \left(m_{2}, n\right)\right)$ and $\left(d_{1}, d_{2}\right)=$ $\left(\min \left(m_{1}, n\right),\left(n-m_{1}\right)^{+}\right)$lie on the line $R_{1}+R_{2}=n(T-n)$. 
Therefore this line cannot intersect with the boundary of $\mathcal{R}_{\mathrm{col}}$ in any other point.

\section{APPENDIX}

Proof of Lemma 1: We know that to find the rate region of the $C_{m-M A C}$ channel we should find out the convex hull of union of the following sets of inequalities

$$
\begin{aligned}
R_{i} & \leq \frac{1}{T} I_{m-M A C}\left(X_{i} ; Y \mid X_{i^{c}}\right), \quad i=1,2, \\
R_{1}+R_{2} & \leq \frac{1}{T} I_{m-M A C}\left(X_{1}, X_{2} ; Y\right)
\end{aligned}
$$

for all $\widetilde{P}_{X_{1} X_{2}}\left(x_{1}, x_{2}\right)=\widetilde{P}_{X_{1}}\left(x_{1}\right) \widetilde{P}_{X_{2}}\left(x_{2}\right)$.

Let us write $I_{m-M A C}\left(X_{1}, X_{2} ; Y\right)$ for the channel $C_{m-M A C}$. We will show that it is equal to the same quantity for that $C_{s}-M A C$ channel.

$$
\begin{aligned}
& =\sum_{\substack{x_{1} \in \widetilde{\mathcal{X}}_{1}, x_{2} \in \widetilde{\mathcal{X}}_{2} \\
y \in \widetilde{\mathcal{Y}}}}\left[\widetilde{P}_{Y \mid X_{1} X_{2}}\left(y \mid x_{1}, x_{2}\right) \widetilde{P}_{X_{1}}\left(x_{1}\right) \widetilde{P}_{X_{2}}\left(x_{2}\right)\right. \\
& \left.\quad \log _{2} \frac{\widetilde{P}_{Y \mid X_{1} X_{2}}\left(y \mid x_{1}, x_{2}\right)}{\widetilde{P}_{Y}(y)}\right] .
\end{aligned}
$$

We know that $\widetilde{P}_{Y \mid X_{1} X_{2}}\left(y \mid x_{1}, x_{2}\right)=\widetilde{P}_{Y \mid X_{1} X_{2}}\left(y \mid x_{1}^{\prime}, x_{2}^{\prime}\right)$ where $\left\langle x_{i}\right\rangle=\left\langle x_{i}^{\prime}\right\rangle$ for $i=1,2$. So with an abuse of notation, we can write the mutual information as

$$
\begin{gathered}
\sum_{\substack{\pi_{1} \in \mathcal{X}_{1}, \pi_{2} \in \mathcal{X}_{2} \\
y \in \widetilde{\mathcal{Y}}}}\left[\widetilde{P}_{Y \mid X_{1} X_{2}}\left(y \mid \pi_{1}, \pi_{2}\right) P_{X_{1}}\left(\pi_{1}\right) P_{X_{2}}\left(\pi_{2}\right)\right. \\
\left.\log _{2} \frac{\widetilde{P}_{Y \mid X_{1} X_{2}}\left(y \mid \pi_{1}, \pi_{2}\right)}{\widetilde{P}_{Y}(y)}\right],
\end{gathered}
$$

where for $i=1,2$, we have $P_{X_{i}}\left(\pi_{i}\right)$ $\sum_{x_{i} \in \widetilde{\mathcal{X}}_{i}:\left\langle x_{i}\right\rangle=\pi_{i}} \widetilde{P}_{X_{i}}\left(x_{i}\right) \quad$ and $\widetilde{P}_{Y \mid X_{1} X_{2}}\left(y \mid \pi_{1}, \pi_{2}\right) \triangleq$ $\widetilde{P}_{Y \mid X_{1} X_{2}}\left(y \mid x_{1}, x_{2}\right)$ for some $x_{i} \in \widetilde{\mathcal{X}}_{i}$ such that $\left\langle x_{i}\right\rangle=\pi_{i}$. Then we have

$$
\begin{aligned}
& I_{m-M A C}\left(X_{1}, X_{2} ; Y\right)= \\
= & \sum_{\pi_{1} \in \mathcal{X}_{1}, \pi_{2} \in \mathcal{X}_{2}} P_{X_{1}}\left(\pi_{1}\right) P_{X_{2}}\left(\pi_{2}\right) \sum_{d_{y}=0}^{n} \sum_{\begin{array}{c}
\pi_{y} \in \mathcal{Y} \\
\operatorname{dim}\left(\pi_{y}\right)=d_{y}
\end{array}} \\
& \sum_{\substack{y \in \widetilde{\mathcal{Y}} \\
\langle y\rangle=\pi_{y}}} \widetilde{P}_{Y \mid X_{1} X_{2}}\left(y \mid \pi_{1}, \pi_{2}\right) \log _{2} \frac{\widetilde{P}_{Y \mid X_{1} X_{2}}\left(y \mid \pi_{1}, \pi_{2}\right)}{\widetilde{P}_{Y}(y)} .
\end{aligned}
$$

Again we can use the property of channel transition matrix where for every $\pi_{1}$ and $\pi_{2}$ we have $\widetilde{P}_{Y \mid X_{1} X_{2}}\left(y_{1} \mid \pi_{1}, \pi_{2}\right)=$ $\widetilde{P}_{Y \mid X_{1} X_{2}}\left(y_{2} \mid \pi_{1}, \pi_{2}\right)$ if $\left\langle y_{1}\right\rangle=\left\langle y_{2}\right\rangle$. So we factor the term in front of summation over $y$ and write

$$
\begin{aligned}
& I_{m-M A C}\left(X_{1}, X_{2} ; Y\right)= \\
= & \sum_{\pi_{1} \in \mathcal{X}_{1}, \pi_{2} \in \mathcal{X}_{2}} P_{X_{1}}\left(\pi_{1}\right) P_{X_{2}}\left(\pi_{2}\right) \sum_{d_{y}=0}^{n} \\
& \sum_{\substack{\pi_{y} \in \mathcal{Y} \\
\operatorname{dim}\left(\pi_{y}\right)=d_{y}}} \psi\left(T, n, d_{y}\right) \widetilde{P}_{Y \mid X_{1} X_{2}}\left(y \mid \pi_{1}, \pi_{2}\right) \log _{2} \frac{\widetilde{P}_{Y \mid X_{1} X_{2}}\left(y \mid \pi_{1}, \pi_{2}\right)}{\widetilde{P}_{Y}(y)},
\end{aligned}
$$

for some $y:\langle y\rangle=\pi_{y}$. Defining $P_{Y \mid X_{1} X_{2}}\left(\pi_{y} \mid \pi_{1}, \pi_{2}\right) \triangleq$ $\psi(\operatorname{dim}(\langle y\rangle)) \widetilde{P}_{Y \mid X_{1} X_{2}}\left(y \mid \pi_{1}, \pi_{2}\right)$ where $\pi_{y}=\langle y\rangle$ we have

$$
\begin{aligned}
& I_{m-M A C}\left(X_{1}, X_{2} ; Y\right)= \\
= & \sum_{\pi_{1} \in \mathcal{X}_{1}, \pi_{2} \in \mathcal{X}_{2}} \sum_{\pi_{y} \in \mathcal{Y}} P_{Y \mid X_{1} X_{2}}\left(y \mid \pi_{1}, \pi_{2}\right) \log _{2} \frac{P_{Y \mid X_{1} X_{2}}\left(\pi_{y} \mid \pi_{1}, \pi_{2}\right)}{P_{Y}\left(\pi_{y}\right)} \\
= & I_{s-M A C}\left(X_{1}, X_{2} ; Y\right) .
\end{aligned}
$$

A similar arguments shows the equality between mutual information of the two channels $C_{m-M A C}$ and $C_{s-M A C}$.

Proof of Lemma 3: There are $\mathcal{G}\left(d_{1}, \alpha\right) \doteq q^{\alpha\left(d_{1}-\alpha\right)}$ different choices for the intersection of $\pi_{1}$ and $\pi_{2}$. We have to choose $d_{2}-\alpha$ basis vectors for the rest of the subspace. This can be done in

$$
\begin{aligned}
& \frac{\left(q^{T}-q^{d_{1}}\right)\left(q^{T}-q^{d_{1}+1}\right) \ldots\left(q^{T}-q^{d_{1}+d_{2}-\alpha-1}\right)}{\left(q^{d_{2}}-q^{\alpha}\right)\left(q^{d_{2}}-q^{\alpha+1}\right) \ldots\left(q^{d_{2}}-q^{d_{2}-1}\right)} \\
& \doteq q^{\left(d_{2}-\alpha\right)\left(T-d_{2}\right)}
\end{aligned}
$$

ways.

Proof of Lemma 4: Define $\pi=\pi_{1} \oplus \pi_{2}$, where $\operatorname{dim}(\pi)=$ $\operatorname{dim}\left(\pi_{1}\right)+\operatorname{dim}\left(\pi_{2}\right)-\operatorname{dim}\left(\pi_{1} \cap \pi_{2}\right)=d_{1}+d_{2}-\alpha \triangleq d$. The proof of this lemma is similar to that of Lemma 3, unless we can only choose the last $d_{2}-\alpha$ basis vectors from $\pi$ instead of $\mathbb{F}_{q}^{T}$. Therefore replacing $T$ in Lemma 3 with $d$, we have

$$
a\left(\pi_{1}, \pi_{2}\right) \doteq q^{\alpha\left(d_{1}-\alpha\right)+\left(d_{2}-\alpha\right)\left(d-d_{2}\right)}=q^{d_{2}\left(d_{1}-\alpha\right)} .
$$

\section{REFERENCES}

[1] R. Koetter and F. Kschischang, "Coding for errors and erasures in random network coding", IEEE Transactions on Information Theory, Volume 54, Issue 8, August 2008.

[2] M. Jafari, C. Fragouli, and S. Diggavi, "Code construction for multiple sources network coding", MobiHoc-S3'09, New Orleans, 2009.

[3] D. Silva and F. R. Kschischang, "Using rank-metric codes for error correction in random network coding", IEEE International Symposium on Information Theory, Nice, France, June 2007.

[4] A. Montanari and R. Urbanke, "Coding for network coding", available online : http://arxiv.org/abs/0711.3935/.

[5] D. Silva, F. R. Kschischang, and R. Koetter, "Capacity of Random Network Coding under a Probabilistic Error Model”, July 2008, available online at http://arxiv.org/pdf/0807.1372/.

[6] M. Jafari, C. Fragouli, and S. Diggavi, "Passive topology discovery for network coded systems", Information Theory Workshop, Norway, 2007.

[7] M. Jafari, C. Fragouli, and S. Diggavi, "Noncoherent multisource network coding", IEEE International Symposium on Information Theory, Page(s) 817-821, Canada, Toronto, July 2008.

[8] M. Jafari, S. Mohajer, C. Fragouli, and S.Diggavi, "On the capacity of non-coherent network coding", IEEE International Symposium on Information Theory, Seoul, Korea, 2009.

[9] P. A. Chou, Y. Wu, and K. Jain, "Practical network coding," Allerton Conference, Monticello, IL, October 2003. 